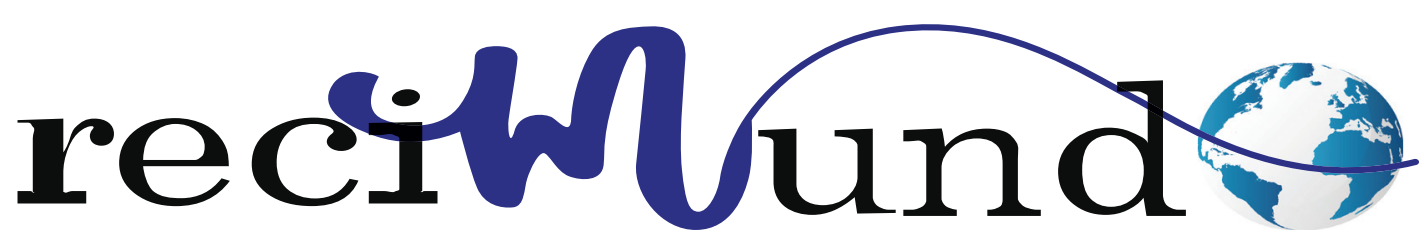

Revista Científica Mundo de la Investigación y el Conocimiento

DOI: 10.26820/recimundo/5.(esp.1).nov.2021.31-42

URL: https://recimundo.com/index.php/es/article/view/1363

EDITORIAL: Saberes del Conocimiento

REVISTA: RECIMUNDO

ISSN: 2588-073X

TIPO DE INVESTIGACIÓN: Artículo de investigación

CÓDIGO UNESCO: 32 Ciencias Médicas

PAGINAS: 31-42

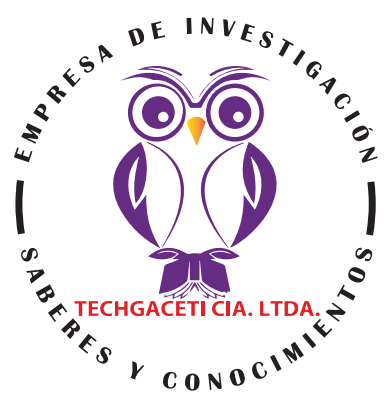

\title{
Enfermedad cerebrovascular isquémica e hipertensión arterial en el hospital Teodoro Maldonado Carbo
}

Ischemic cerebrovascular disease and arterial hypertension at the Teodoro Maldonado Carbo hospital

Doença cerebrovascular isquêmica e hipertensão arterial no hospital Teodoro Maldonado Carbo

\section{Maritza Alexandra Borja Santillán'; Jorge Luis Samaniego Gallino; Sharon Dayana Aguirre Ruilova3; María Gabriela Prieto Ulloa ${ }^{4}$}

RECIBIDO: 15/09/2021 ACEPTADO: 05/10/2021 PUBLICADO: 29/11/2021

1. Doctora en Educación; Magister en Gerencia de Salud para el Desarrollo Local; Especialista en Gerencia y Planificación Estratégica en Salud; Diploma Superior en Desarrollo Local y Salud; Magister en Emergencias Médicas; Medico; Licenciada en Ciencias de la Educación mención Lengua Inglesa y Lingüística; Docente Universidad de Guayaquil, Facultad de Ciencias Médicas, Guayaquil, Ecuador; maritza.borjas@ug.edu.ec; (iD https://orcid.org/0000-0002-2803-4662

2. Médico; Facultad de Ciencias Médicas; Universidad de Guayaquil, Guayaquil, Ecuador; samaniego-j|@hotmail.com; (iD https://orcid.org/0000-0001-9630-8349

3. Médico; Facultad de Ciencias Médicas; Universidad de Guayaquil, Guayaquil, Ecuador; sharon.aguirre1@ gmail.com; (D) https://orcid.org/0000-0002-9355-260X

4. Psicóloga Clínica, Magister en Terapia Familiar Sistémica y de Pareja. Docente de la Facultad de Ciencias Médicas Universidad de Guayaquil; Guayaquil, Ecuador; gabriela.prietou@ug.edu.ec; (D) https://orcid.org/00000002-5574-7633

\section{CORRESPONDENCIA}

Maritza Alexandra Borja Santillán

maritza.borjas@ug.edu.ec

Guayaquil, Ecuador 


\section{RESUMEN}

La enfermedad cerebrovascular isquémica es una de las principales causas de mortalidad a nivel mundial y se define como la muerte del tejido cerebral ocasionada por la obstrucción arterial que limita el aporte sanguíneo. El presente trabajo de investigación se realizó en el Hospital Teodoro Maldonado Carbo de la ciudad de Guayaquil, cuyo objetivo fue determinar la relación que existe entre la enfermedad cerebrovascular isquémica y la hipertensión arterial en los pacientes mayores de 40 años de enero del 2016 a diciembre del 2020. Metodología: Esta investigación es de tipo retrospectivo, correlacional, con enfoque cuantitativo, diseño de investigación no experimental y corte transversal. Tiene como universo a todos los pacientes mayores de 40 años con diagnóstico de enfermedad cerebrovascular isquémica e hipertensión arterial atendidos en el Hospital Teodoro Maldonado Carbo de enero del 2016 a diciembre del 2020 y que tenían sus historias clínicas completas, es decir, 476 pacientes; la muestra se convirtió en todos los pacientes del universo que cumplen con los criterios de inclusión y exclusión anteriormente mencionados. Resultados: Del total de la muestra el 63,2\% corresponden al sexo masculino; el rango de edad predominante fue de 71 a 80 años con el 32,8\%; y el 37,2\% de los pacientes presentaron hipertensión arterial grado 1. Se determinó la asociación entre la enfermedad cerebrovascular isquémica y los grados de hipertensión arterial evidenciando una asociación significativa (P-VALOR=0,001<0,05).

Palabras clave: Enfermedad, infarto, isquemia, hipertensión, hospital.

\section{ABSTRACT}

Ischemic cerebrovascular disease is one of the leading causes of mortality worldwide and is define as the death of brain tissue caused by arterial obstruction that limits blood supply. The present research work was carried out at the Teodoro Maldonado Carbo Hospital in the city of Guayaquil, whose objective was to determine the relationship between ischemic cerebrovascular disease and arterial hypertension in patients over 40 years of age from January 2016 to December 2020. Methodology: This research is retrospective, correlational, with a quantitative approach, non-experimental research design and cross section. Its universe is all patients over 40 years of age with a diagnosis of ischemic cerebrovascular disease and arterial hypertension treated at the Teodoro Maldonado Carbo Hospital from January 2016 to December 2020 and who had their complete medical records, that is, 476 patients; the sample was converted into all the patients in the universe who meet the aforementioned inclusion and exclusion criteria. Results: Of the total sample, $63.2 \%$ correspond to the male sex; the predominant age range was from 71 to 80 years and $37.2 \%$ of the patients had grade 1 hypertension. The association between ischemic cerebrovascular disease and grades of hypertension was determined, showing a significant association $(P-V A L U E=0.001<0,05)$.

Keywords: Disease, infarct, ischemic, hypertension, hospital.

\section{RESUMO}

A doença cerebrovascular isquêmica é uma das principais causas de mortalidade em todo o mundo e é definida como a morte do tecido cerebral causada por obstrução arterial que limita o fornecimento de sangue. O presente trabalho de pesquisa foi realizado no Hospital Teodoro Maldonado Carbo, na cidade de Guayaquil, cujo objetivo foi determinar a relação entre doença cerebrovascular isquêmica e hipertensão arterial em pacientes com mais de 40 anos de idade no período de janeiro de 2016 a dezembro de 2020. Metodologia: a pesquisa é retrospectiva, correlacional, com abordagem quantitativa, desenho de pesquisa não experimental e corte transversal. Seu universo são todos os pacientes com mais de 40 anos com diagnóstico de doença cerebrovascular isquêmica e hipertensão arterial atendidos no Hospital Teodoro Maldonado Carbo de janeiro de 2016 a dezembro de 2020 e que apresentassem prontuário completo, ou seja, 476 pacientes; a amostra foi convertida em todos os pacientes do universo que atendessem aos critérios de inclusão e exclusão supracitados. Resultados: Do total da amostra, 63,2\% correspondem ao sexo masculino; a faixa etária predominante foi de 71 a 80 anos e 37,2\% dos pacientes apresentavam hipertensão grau 1. A associação entre doença cerebrovascular isquêmica e graus de hipertensão foi determinada, mostrando uma associação significativa (P-VALUE $=0,001<0,05$ ).

Palavras-chave: Doença, infarto, isquemia, hipertensão, hospital. 


\section{Introducción}

La Enfermedad Vascular Cerebral, es la consecuencia de múltiples eventos patológicos que lesionan la vasculatura del sistema nervioso provocando de esta manera la deficiencia de oxígeno en los tejidos cerebrales lo que ocasiona isquemia y a su vez alteración neuronal, suelen presentarse con una amplia gama de síndromes, cada una con sus tipologías particulares que los caracteriza. La enfermedad cerebrovascular es la protagonista de importantes cifras de mortalidad en todo el mundo; se cree que por cada infarto que presenta síntomas hay 9 que se producen de manera silenciosa y que afectan el nivel cognitivo de quienes la padecen; esta patología se presenta en cualquier etapa de la vida, sin embargo, es más común encontrarla en mayores de 65 años y la probabilidad de presentarla se duplica por cada década a partir de los 55 años.

Hoy en día existen grandes avances en lo que respecta al conocimiento de la fisiopatología y los factores de riesgo de la enfermedad cerebrovascular, a pesar de ello, en países desarrollados los cálculos estadísticos hacen notorio que la incidencia de esta enfermedad ha permanecido constante, suceso que se puede explicar por el incremento en las expectativas de vida y el aumento de esta patología en el sexo femenino. Dentro de los factores que ponen en riesgo el aumento de la incidencia y prevalencia de esta enfermedad y que tiene especial relevancia es la hipertensión arterial, ya que durante los últimos siglos la sociedad experimenta variaciones significativas en los estilos de vida y en los hábitos alimenticios, que, probablemente sean los causantes del aumento en los niveles de la presión arterial, por ende, se considera a la hipertensión arterial como una enfermedad crónica de gran importancia como problema de salud pública. (Ministerio de Salud Gobierno Entre Ríos, 2017)
La Organización Mundial de la Salud considera a la enfermedad cerebrovascular como la tercera causa de mortalidad a nivel mundial; luego de las enfermedades cardiacas y el cáncer, generalmente acontece en países de bajos recursos, lo que se traduce con el $10 \%$ de personas fallecidas; tiene una mayor incidencia en el sexo masculino, sin embargo, al presentarse en el sexo femenino la enfermedad aumenta su mortalidad, esto debido a que suelen presentarse en una edad mucho más avanzada en relación a los hombres, por lo que existen más probabilidades de que las mujeres no sobrevivan, partiendo de ello, la Organización Mundial de la Salud también nos menciona que en el año 2015 se presentaron más de 6 millones de fallecimientos a causa de la enfermedad cerebrovascular en todo el mundo, de los cuales 3'250.217 son mujeres y 2'990.394 pertenecen al sexo masculino. (Ministerio de Salud Gobierno Entre Ríos, 2017) (Busto, 2019)

Por otro lado, en Ecuador, actualmente esta enfermedad representa el primer lugar como causante de mortalidad, mientras que en 1975 aún ocupaba el noveno lugar, sin embargo, 25 años más tarde; es decir, en 1990, se posicionó como la primera causa, dato que aún se sigue manteniendo hasta la presente fecha debido a que el total de fallecimientos en los últimos 25 años es de 1'410.986 y obviando las causas no definidas se obtuvo el número de 1'222.173 muertes, representando el 77.897, es decir, $6,70 \%$ de los fallecimientos, seguida de la influenza y neumonía con un 73.441 dicho en porcentaje, $6,32 \%$ y diabetes mellitus con un 70.536, es decir, 6.07\% ubicándose en el tercer lugar; mientras que el cuarto lugar lo ocupan las enfermedades hipertensivas con un 67.155, es decir 5,78\%, quinto lugar las enfermedades isquémicas del corazón con 61.759 , dicho en porcentaje $5,31 \%$ y finalmente el sexto lugar los accidentes de transporte terrestre con 60.033 que representa el $5,16 \%$ de fallecimientos. (Moreno-Zambrano, Santamaría, \& Ludeña, 2017)

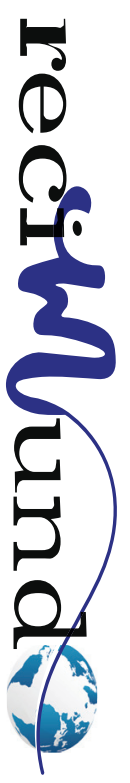


En el Hospital Teodoro Maldonado Carbo hay una incidencia importante de personas hipertensas y de enfermedad cerebrovascular, situación agravada en los últimos años por la crisis sanitaria con tendencia a incrementarse y del cual no existen estudios que demuestren información estadística de manera específica y actualizada sobre la prevalencia de estas patologías, por lo que se ha convertido en nuestro escenario de enfoque. Si bien es cierto ya existen publicaciones a nivel mundial sobre enfermedad cerebrovascular, estudios que abarcan tanto factores de riesgo, epidemiología, manejo clínico y medidas terapéuticas, mientras que a nivel local; hay trabajos que sí relacionan la enfermedad cerebrovascular isquémica inclusive con la hipertensión arterial directamente en hospitales de la ciudad de Guayaquil, pero la información presentada es limitada a un periodo corto de tiempo y ya no es actualizada, además que la relación planteada no se ha realizado en el establecimiento de salud en estudio, sumado a esto el enfoque preventivo como recurso que se le otorga a nuestro estudio le brinda un plus importante que lamentablemente no ha sido explotado.

La conveniencia de esta investigación está justificada porque proporciona información actualizada del comportamiento demográfico, severidad de la enfermedad cerebrovascular isquémica y su relación con la hipertensión arterial en la población de pacientes del Hospital Teodoro Maldonado Carbo. Es necesario conocer el grado de severidad de la enfermedad cerebrovascular isquémica y su relación con la hipertensión arterial a fin de que esos resultados permitan a los profesionales de salud la toma de decisiones para reducir la incidencia de complicaciones o secuelas, afianzar conocimientos y ponerlos en práctica y a su vez, como aporte a la sociedad al brindar información clave enfocada a la prevención de este cuadro mediante la utilización de materiales didácticos, por medio de los cuales, podamos aportar a la sociedad y a la comunidad médica.
El objetivo del presente estudio es determinar la relación que existe entre la enfermedad cerebrovascular isquémica y la hipertensión arterial en los pacientes mayores de 40 años atendidos en el Hospital Teodoro Maldonado Carbo.

\section{Metodología}

Para el presente trabajo de investigación se empleó una metodología cuantitativa.

\section{Tipo de investigación}

Retrospectivo, diseño de investigación no experimental y corte transversal.

\section{Métodos de investigación}

Como método de investigación empírica utiliza la observación y como método de investigación teórica, es de tipo analítico.

\section{Universo}

El universo de estudio corresponde a los pacientes mayores de 40 años con el diagnóstico de enfermedad cerebrovascular isquémica e hipertensión arterial que fueron atendidos en el Hospital Teodoro Maldonado Carbo del 1 de enero del 2016 al 31 de diciembre del 2020 y que corresponde a 476 pacientes.

\section{Muestra}

La muestra se convirtió en todos los pacientes del universo que cumplen con los criterios de inclusión y exclusión.

\section{Instrumentos de recolección de datos}

Los datos se recolectaron a través de una hoja de cálculo del programa Microsoft Excel 2013 y los datos fueron extraídos de las historias clínicas de los pacientes que cumplen con los criterios de la investigación.

\section{Procesamiento de los datos}

El procesamiento de datos se lo realizó mediante el software de IBM, SPSS 25, en el cual se procedió a la introducción de datos de cada uno de los individuos que fueron seleccionados para el estudio y la realiza- 
ción de los análisis estadísticos pertinentes, además de la creación de tablas, gráficas y porcentajes necesarios.

\section{Consideraciones bioéticas}

Se llevó a cabo este trabajo de investigación teniendo como base los 4 principios de la ética en investigación biomédica: principio de autonomía, principio de beneficencia, principio de no maleficencia y principio de justicia. Con el objetivo de mantener la confidencialidad de los registros médicos y datos personales de los pacientes que fueron utilizados en este trabajo de investigación.

\section{Resultados}

Se presenta el análisis descriptivo e inferencial de los 476 pacientes mayores de
40 años con enfermedad cerebrovascular isquémica e hipertensión arterial, atendidos en el servicio de hospitalización del Hospital Teodoro Maldonado Carbo en el período 2016-2020. La descripción de las variables se hace mediante tablas de frecuencias absolutas y porcentuales, así como gráficos de barras permiten explorar los datos. También se muestra el análisis inferencial para el contraste de la prueba de hipótesis de la investigación utilizando un nivel de significancia del $5 \%$.

\section{Análisis descriptivo}

Objetivo: Establecer el número de pacientes con diagnóstico de enfermedad cerebrovascular isquémica que presentaron hipertensión arterial y que fueron atendidos en el Hospital Teodoro Maldonado Carbo de enero del 2016 a diciembre del 2020.

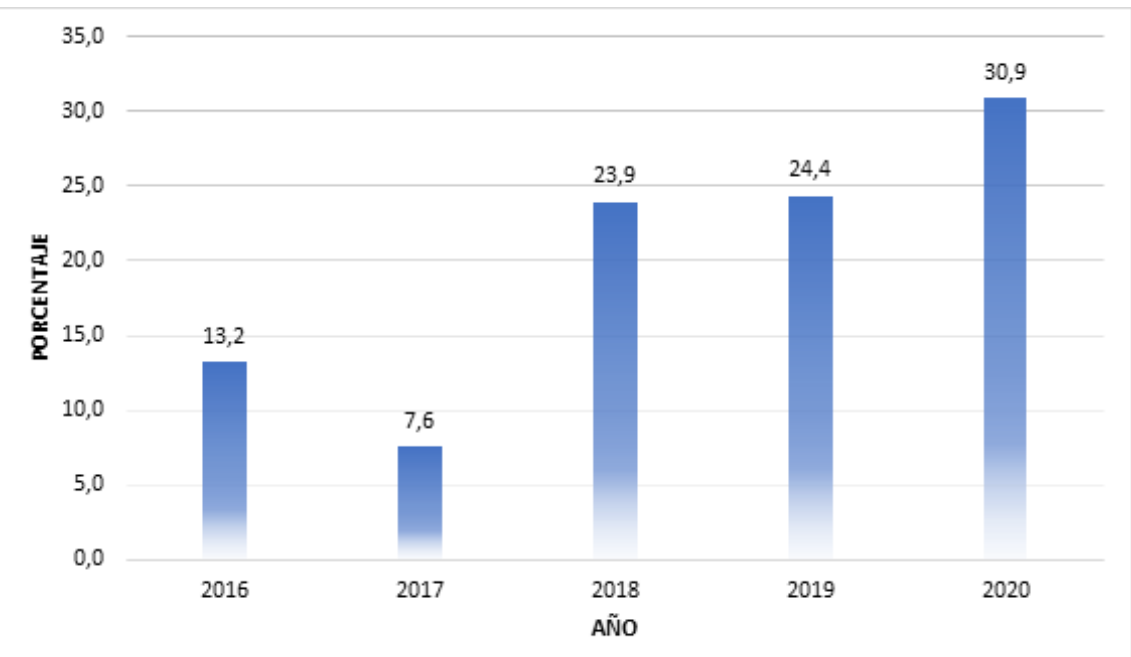

Figura 1. Gráfico de barras de la distribución de pacientes atendidos en el Hospital Teodoro Maldonado Carbo en el período 2016-2020.

Nota: Las barras representan el porcentaje de pacientes atendidos en el hospital de acuerdo con el año, permite ver el crecimiento durante el período en estudio. Elaboración propia. Fuente de datos: Historias Clínicas AS400 (sistema interno del Hospital)

Fuente: Investigación

Análisis: Se exhibe la cantidad de pacientes atendidos durante el período 2016 - 2020. Se observó un aumento a partir del año 2017 de 16.3\% para el año 2018, continuando en crecimiento, pero menos acelerado. Sin embargo, si observamos el año 2016 y lo comparamos con el año 2020 hubo un crecimiento mayor al 50\%. Así mismo el año 2020 mostró la mayor afluencia de pacientes atendidos (30,9\%), seguido del año $2019(24,4 \%$ ) y el año 2018 (23.9\%), juntos representan el 79,9\% de los pacientes atendidos durante el período en estudio. 


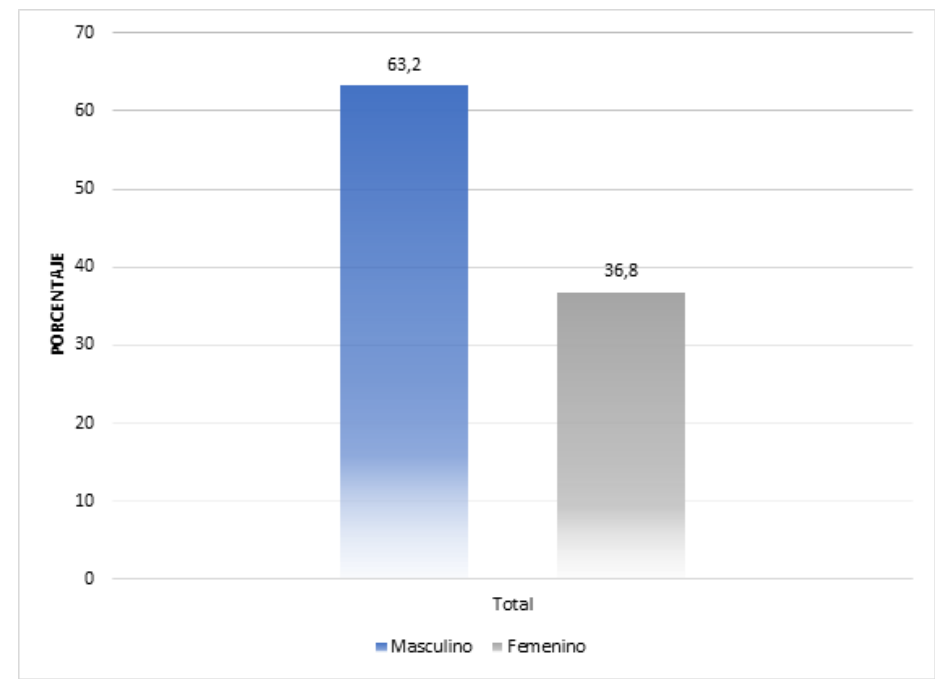

Figura 2. Gráfico de barras del sexo de los pacientes atendidos en el Hospital Teodoro Maldonado Carbo en el período 2016-2020.

Nota: Las barras representan el porcentaje de pacientes agrupados por sexo, atendidos en el hospital. Elaboración propia. Fuente de datos: Historias Clínicas AS400 (sistema interno del Hospital)

Fuente: Investigación

Análisis: Entre las características demográficas más relevantes de los pacientes, se puede observar en la figura 2 que el 63,2\% representan el sexo masculino mientras que el 36,8 al sexo femenino. En cambio, en la tabla 3 se muestra un aumento progresivo a través del período en estudio.

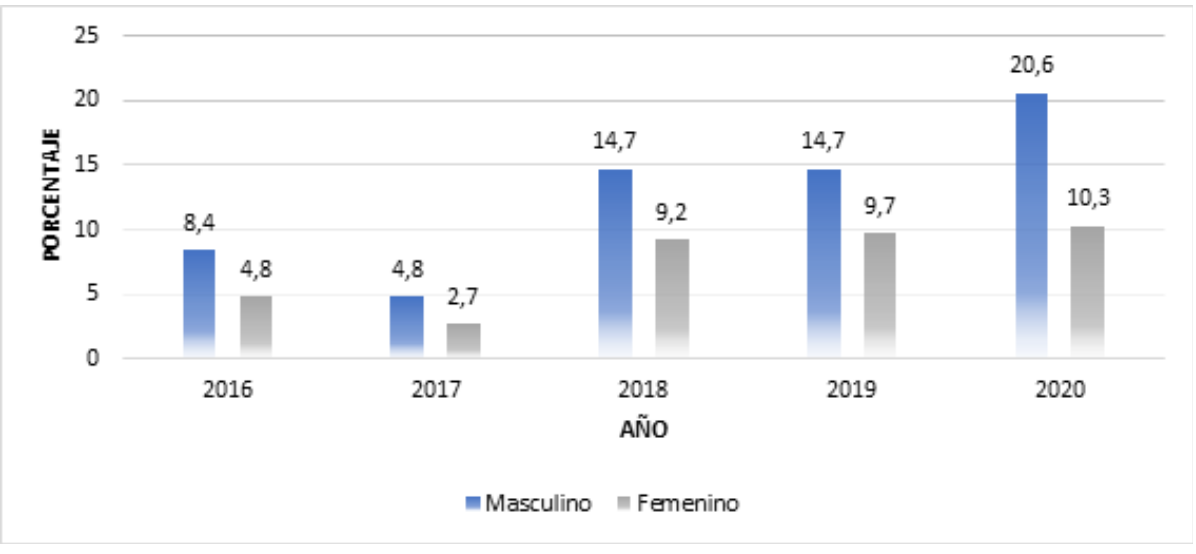

Figura 3. Gráfico de barras por año del sexo de los pacientes atendidos en el Hospital Teodoro Maldonado Carbo en el período 2016-2020.

Nota: Las barras representan el porcentaje de pacientes agrupados por sexo, atendidos en el hospital, permite ver los grupos con mayor cantidad de pacientes. Elaboración propia. Fuente de datos: Historias Clínicas AS400 (sistema interno del Hospital).

Fuente: Investigación 


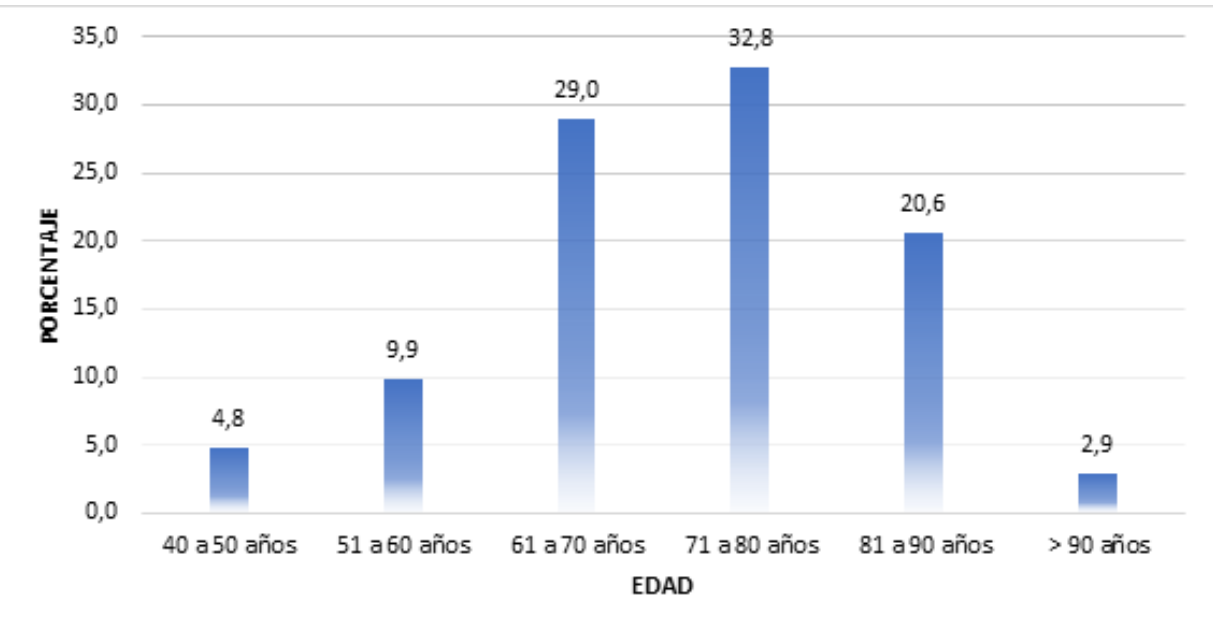

Figura 4. Gráfico de barras de la edad de pacientes atendidos en el Hospital Teodoro Maldonado Carbo en el período 2016-2020.

Nota: Las barras representan el porcentaje de pacientes en cada grupo etario, atendidos en el hospital, permite ver los grupos con mayor cantidad de pacientes. Elaboración propia. Fuente de datos: Historias Clínicas AS400 (sistema interno del Hospital)

Fuente: Investigación

Análisis: En la figura 4, se exponen los grupos etarios con mayor cantidad de pacientes fueron de 61 a 70 años (29\%), de 71 a 80 años (32,8\%) y de 81 a 90 años (20,6\%), de igual forma el grupo etario con menor cantidad de pacientes fueron los mayores a 90 años (2,9\%), y el grupo etario de 40 a 60 años representan el 14,7\%.

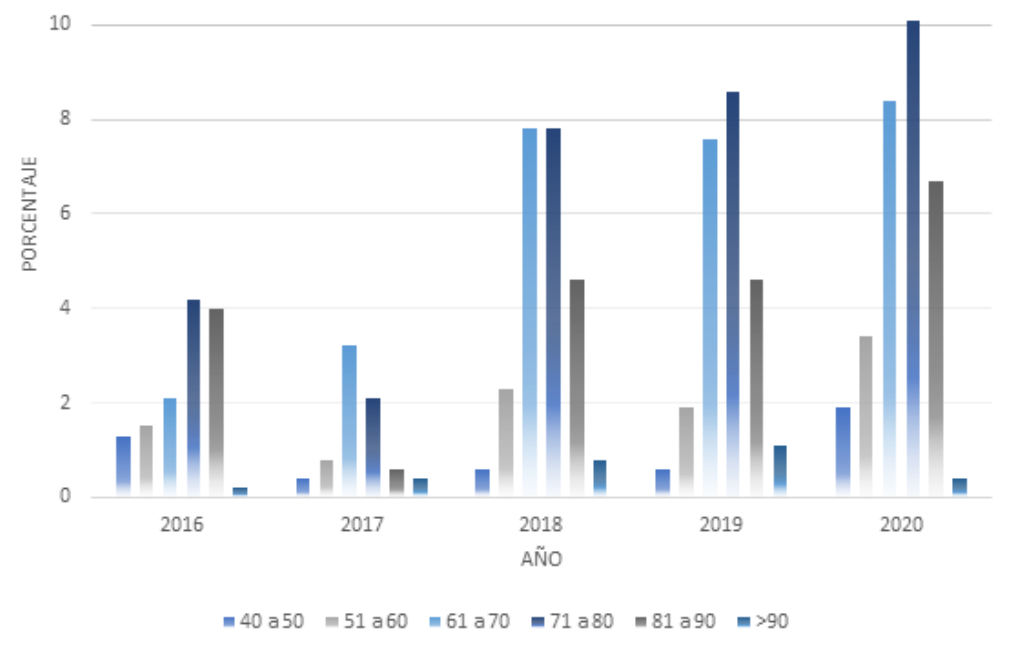

Figura 5. Gráfico de barras de la edad de pacientes atendidos en el Hospital Teodoro Maldonado Carbo en el período 2016-2020.

Nota: Las barras representan el porcentaje de pacientes en cada grupo etario, atendidos en el hospital, permite ver los grupos con mayor cantidad de pacientes. Elaboración propia. Fuente de datos: Historias Clínicas AS400 (sistema interno del Hospital). 
Fuente: Investigación

Análisis: En la figura 5 se detallan los grupos etarios distribuidos por año, donde se observa la incidencia de casos en cada periodo de tiempo.

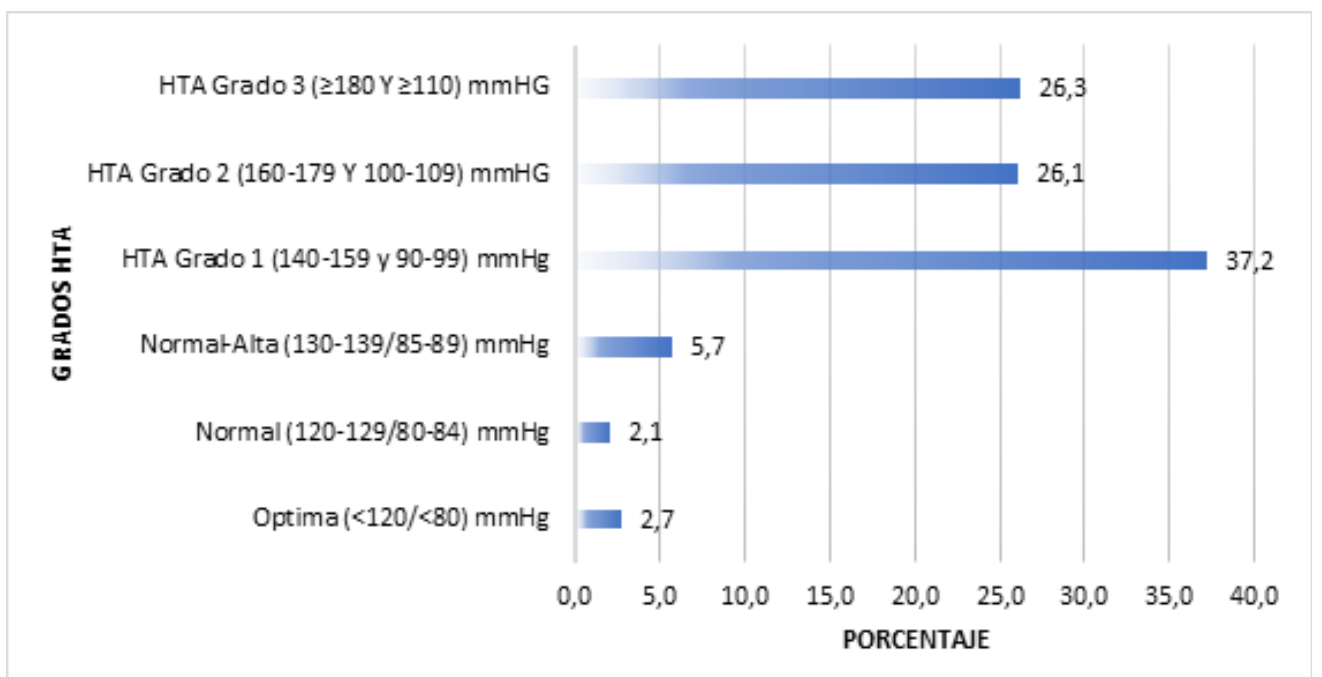

Figura 6. Gráfico de barras del grado de hipertensión arterial de pacientes atendidos en el Hospital Teodoro Maldonado Carbo en el período 2016-2020.0.

Nota: Las barras representan el porcentaje de pacientes clasificados según el grado de hipertensión arterial, atendidos en el hospital, permite ver los grados con mayor cantidad de pacientes. Elaboración propia. Fuente de datos: Historias Clínicas AS400 (sistema interno del Hospital).

Fuente: Investigación

Análisis: De la figura 6, según el grado de hipertensión, el 37,2\% de los pacientes presentaron Grado 1, el 26,3\% y 26,1\% Grado 3 y Grado 2, respectivamente, que representan el $89,6 \%$ del total.

Objetivo. Correlacionar los tipos de enfermedad cerebrovascular isquémica y de hipertensión arterial en pacientes con diagnóstico de enfermedad cerebrovascular isquémica atendidos en el Hospital Teodoro Maldonado Carbo de enero del 2016 a diciembre del 2020.

Se determina la asociación entre los grados de hipertensión arterial en pacientes con diagnóstico de enfermedad cerebrovascular isquémica que presentaron hipertensión arterial. Se utilizó la distribución Chi-cuadrado. Los resultados se muestran en la tabla 13, donde se observaron asociaciones sig- nificativas entre los grados de hipertensión arterial y el diagnóstico según CIE10 (p-valor $=0,001<0,05)$. 
Tabla 1. Asociación de enfermedad cerebrovascular isquémica con el grado de hipertensión arterial.

\begin{tabular}{|c|c|c|c|c|c|c|c|}
\hline \multirow[b]{2}{*}{ Variables } & \multicolumn{6}{|c|}{ Grados de HTA } & \multirow[b]{2}{*}{ P-valor } \\
\hline & Optima & Normal & $\begin{array}{l}\text { Normal- } \\
\text { Alta }\end{array}$ & $\begin{array}{c}\text { HTA } \\
\text { Grado } 1\end{array}$ & $\begin{array}{c}\text { HTA } \\
\text { Grado } 2\end{array}$ & $\begin{array}{c}\text { HTA } \\
\text { Grado } 3\end{array}$ & \\
\hline \multicolumn{8}{|l|}{ CIE10 } \\
\hline $\begin{array}{lr}\text { Accidente cerebral } \\
\text { encefálico agudo, no } \\
\text { especificado } \\
\text { hemorrágico } \\
\text { isquémico }\end{array}$ & $0,8 \%$ & $0,4 \%$ & $1,3 \%$ & $9,0 \%$ & $7,4 \%$ & $9,0 \%$ & $0,001^{*}$ \\
\hline $\begin{array}{l}\text { Infarto cerebral } \\
\text { debido a embolia de } \\
\text { arterias cerebrales }\end{array}$ & $0 \%$ & $0 \%$ & $0 \%$ & $0 \%$ & $0,2 \%$ & $0 \%$ & \\
\hline $\begin{array}{l}\text { Infarto cerebral } \\
\text { debido a embolia de } \\
\text { arterias } \\
\text { precerebrales }\end{array}$ & $0 \%$ & $0,4 \%$ & $0 \%$ & $0,4 \%$ & $0,4 \%$ & $0,2 \%$ & \\
\hline $\begin{array}{l}\text { Infarto cerebral } \\
\text { debido a oclusión o } \\
\text { estenosis no } \\
\text { especificada de } \\
\text { arterias cerebrales }\end{array}$ & $0 \%$ & $0,2 \%$ & $0 \%$ & $0 \%$ & $0,2 \%$ & $0 \%$ & \\
\hline $\begin{array}{l}\text { Infarto cerebral } \\
\text { debido a trombosis } \\
\text { de arterias }\end{array}$ & $0 \%$ & $0 \%$ & $0 \%$ & $1,1 \%$ & $0,4 \%$ & $0,6 \%$ & \\
\hline 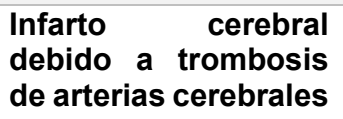 & $0 \%$ & $0,4 \%$ & $0 \%$ & $1,1 \%$ & $1,1 \%$ & $0,6 \%$ & \\
\hline $\begin{array}{l}\text { Infarto cerebral no } \\
\text { especificado }\end{array}$ & $0,6 \%$ & $0,4 \%$ & $2,1 \%$ & $16,6 \%$ & $11,1 \%$ & $5,7 \%$ & \\
\hline $\begin{array}{l}\text { Isquemia cerebral } \\
\text { transitoria, sin otra } \\
\text { especificación }\end{array}$ & $0 \%$ & $0 \%$ & $0,6 \%$ & $1,3 \%$ & $0,4 \%$ & $1,1 \%$ & \\
\hline $\begin{array}{l}\text { Oclusión y estenosis } \\
\text { de arteria precerebral } \\
\text { no especificada }\end{array}$ & $0 \%$ & $0 \%$ & $0 \%$ & $0,2 \%$ & $0 \%$ & $0 \%$ & \\
\hline $\begin{array}{l}\text { Otras enfermedades } \\
\text { cerebrovasculares }\end{array}$ & $1,1 \%$ & $0,2 \%$ & $1,3 \%$ & $7,4 \%$ & $4,4 \%$ & $8,2 \%$ & \\
\hline $\begin{array}{l}\text { Otros } \\
\text { cerebrales }\end{array}$ & $0,2 \%$ & $0 \%$ & $0 \%$ & $0,2 \%$ & $0 \%$ & $0,4 \%$ & \\
\hline $\begin{array}{lr}\text { Secuelas } & \text { de } \\
\text { accidente } & \text { vascular } \\
\text { encefálico } & \end{array}$ & $0 \%$ & $0 \%$ & $0,2 \%$ & $0 \%$ & $0,4 \%$ & $0,2 \%$ & \\
\hline $\begin{array}{l}\text { Síndromes } \\
\text { vasculares } \\
\text { encefálicos en enf } \\
\text { cerebrovasculares }\end{array}$ & $0 \%$ & $0 \%$ & $0,2 \%$ & $0 \%$ & $0 \%$ & $0,2 \%$ & \\
\hline
\end{tabular}

Nota: Los grados de HTA son Optima $(120 /<80)$ mmHg, Normal $(120-129 / 80-84)$ mmHg, Normal-Alta (130-139/85-89) mmHg, Grado 1 (140-159 y 90-99) mmHg, Grado 2 (160-179 Y 100-109) mmHG y Grado 3 ( $\geq 180$ Y $\geq 110)$ mmHG. * Valores significativos al 5\%. Elaboración propia. Fuente de datos: Historias Clínicas AS400 (sistema interno del Hospital)

Fuente: Investigación 


\section{Discusión}

El número de pacientes con diagnóstico de enfermedad cerebrovascular isquémica que presentaron hipertensión arterial y que fueron atendidos en el Hospital Teodoro Maldonado Carbo de enero del 2016 a diciembre del 2020 fue de 476, donde se realizó una limitación por año en la cual se observó un aumento a partir del año 2017 de $16.3 \%$ para el año 2018, continuando en crecimiento, pero menos acelerado. Sin embargo, si observamos el año 2016 y lo comparamos con el año 2020 hubo un crecimiento mayor al 50\%. Así mismo el año 2020 mostró la mayor afluencia de pacientes atendidos $(30,9 \%)$, seguido del año 2019 (24,4\%) y el año 2018 (23.9\%), juntos representan el $79,9 \%$ de los pacientes atendidos durante el período en estudio, estos son parámetros que ningún otro estudio realiza comparación.

Con respecto al sexo, en el presente estudio refleja que el $63,2 \%$ representan al sexo masculino, similar a los resultados expuestos por (Venegas Rodríguez, Hernández Pedroso, \& Chacón Montano, 2020) donde predominó el sexo masculino con $61,5 \%$, al igual que (Piloto Cruz, Suarez Rivero, Belaunde Clausell, \& Castro Jorge, 2020) con el 58,7 \%, (Bernarda Liliam Reino Pintado, Federico David Pacheco Borjas, Silvana Valeria Pucha Pucha, Silvana Estefanía Ordóñez Gavilanez, Gabriel Guillermo Enríquez Carvajal, Viviana Estefania Piedra Aguirre,Daniel Efraín Parra Coronel, Marcos Palacio Rojas, 2018) con el 63,6\% y (Fernández Siguencia \& Solís Pillaga, 2019) con el 52\%. En relación al grupo etario, nuestro estudio presenta una prevalencia de estas patologías en el rango de 71 a 80 años (32,8\%) en primer lugar y en segundo 61 a 70 años (29\%), resultados que muestran relación a los propuestos por Venegas Rodríguez Rafael, et al donde la edad de 65 a 74 años (40\%) predomina, al igual que Anabel Piloto Cruz, et al que obtuvo como resultado que el $54,7 \%$ de los pacientes eran mayores de 70 años, Mónica del Rocío Fernán- dez Siguencia, et al que presenta un $72 \%$ en adultos mayores de 64 años, Bernarda Liliam Reino Pintado, et al presenta la edad promedio de $60 \pm 9$ años y (Sepúlveda-Contreras, 2020) en su artículo donde denota un fuerte incremento en la población sobre 51 años.

En nuestro estudio, la gravedad de enfermedad cerebrovascular isquémica en los pacientes atendidos en el Hospital Teodoro Maldonado Carbo de enero del 2016 a diciembre del 2020 fue establecida mediante la escala de NIHSS donde la mayoría de los pacientes se encontró en el nivel moderado $(64,1 \%)$, se observa que los pacientes que tienen un grado de enfermedad entre grave (16-24 puntos) y muy grave ( $\geq 25$ puntos) tienen mayor riesgo de fallecer y la mortalidad total fue de $21.6 \%$, coincidiendo con los resultados propuestos por Venegas Rodríguez Rafael, et al en que un índice de NIHSS elevado se asoció a una evolución no satisfactoria más no se relaciona en que el $82,7 \%$ de su población estudiada obtuvo un índice de NIHSS menor de 20 puntos y la letalidad total fue del $10,0 \%$.

Se comparte el criterio con los demás autores de que la hipertensión arterial es un factor de riesgo que se asocia con la enfermedad cerebrovascular y de manera significativa con la de tipo isquémica, que en conjunto con los demás factores de riesgo tanto modificables y no modificables, juegan un papel fundamental en la prevención, curso y progresión de la enfermedad, considerada un verdadero problema en la saIud pública mundial, además que se deben escatimar esfuerzos velando por un sistema sanitario de calidad y sociedades más comprometidas.

\section{Conclusiones}

El trabajo de investigación realizado en el Hospital Teodoro Maldonado Carbo, concluye en lo siguiente:

- Se observó una cantidad significativa de pacientes atendidos a lo largo de cinco 
años que muestra la realidad de no sólo la ciudad de Guayaquil, sino a su vez del país, ya que muchos de estos pacientes son referidos de otras ciudades, evidenciando un aumento a partir del año 2017 de $16.3 \%$ para el año 2018, continuando en crecimiento, pero menos acelerado, sin embargo, si se compara el año 2016 con el año 2020 hubo un crecimiento mayor al $50 \%$.

- Se evidenciaron las características demográficas como son la edad y sexo de la población, donde el 63,2\% representan el sexo masculino y el grupo etario con mayor cantidad de pacientes fueron los ubicados en el rango de 61 a 80 años representando el $61,8 \%$ del total de pacientes atendidos.

- Se categorizaron los pacientes según los grados de hipertensión permitiendo establecer su incidencia en la comunidad y cuan arraigada esta se encuentra, donde se identificó que el 89,6\% de los pacientes atendidos presentaron un cuadro de hipertensión arterial, en el cual el $37,2 \%$ de los pacientes presentaron hipertensión arterial Grado 1.

- Se determinó la asociación significativa entre enfermedad cerebrovascular isquémica e hipertensión arterial en los pacientes atendidos en el Hospital Teodoro Maldonado Carbo y la importancia entre ambas en relación a los demás factores de riesgo.

Se concluye que existe una correlación entre el factor de riesgo modificable que es la hipertensión arterial en desencadenar un accidente cerebro vascular isquémico. Además, se determina que se asocian otros factores de riesgo tales como la edad, el tiempo de atención y la severidad de la enfermedad cerebrovascular isquémica con la mortalidad de los pacientes estudiados en el Hospital Teodoro Maldonado Carbo.

\section{Bibliografía}

Bernarda Liliam Reino Pintado, Federico David Pacheco Borjas, Silvana Valeria Pucha Pucha, Silvana Estefanía Ordóñez Gavilanez, Gabriel Guillermo Enríquez Carvajal, Viviana Estefania Piedra Aguirre,Daniel Efraín Parra Coronel, Marcos Palacio Rojas. (2018). Factores asociados a enfermedad cerebrovascular en pacientes que acuden al Hospital de Especialidades Dr. Abel Gilbert Pontón, Guayaquil. Archivos Venezolanos de Farmacología y Terapéutica, 37(3), 270-274.

Busto, J. E. (Septiembre de 2019). Las enfermedades cerebrovasculares como problema de salud - Revista Cubana de Neurología y Neurocirugía, $9(2)$.

Fernández Siguencia, M., \& Solís Pillaga, P. (2019). Incidencia de accidente cerebrovascular isquémico en pacientes mayores a 50 años que padecen hipertensión arterial en el Hospital José Carrasco Arteaga, Cuenca, diagnosticada mediante tomografía computarizada en el período Enero-Junio 2019. Cuenca, Ecuador: Universidad de Cuenca. Recuperado el 2021, de Repositorio Universidad de Cuenca-Facultad de Ciencias Médicas.

Ministerio de Salud Gobierno Entre Ríos. (2017). Recuperado el Julio de 2021, de Boletín N 12 Enfermedades Crónicas No Transmisibles: http://www. afam.org.ar/textos/10_05_2017/las_enfermedades_cronicas_no_transmisibles.pdf

Moreno-Zambrano, D., Santamaría, D., \& Ludeña, C. (2017). Enfermedad Cerebrovascular en el Ecuador: Análisis de los Últimos 25 años de mortalidad, realidad actual y recomendaciones. Revista Ecuatoriana de Neurología, 25(1-3), 17-20. Obtenido de http://revecuatneurol.com/wp-content/uploads/2017/05/Enfermedad-cerebrovascular-ecuador-analisis-mortalidad.pdf

Piloto Cruz, A., Suarez Rivero, B., Belaunde ClauseII, A., \& Castro Jorge, M. (2020). La enfermedad cerebrovascular y sus factores de riesgo. Revista Cubana de Medicina Militar, 49(3), e0200568.

Sepúlveda-Contreras, J. (2020). Caracterización de pacientes con accidente cerebrovascular ingresados en un hospital de baja complejidad en Chile. Universidad y Salud, 23(1), 8-12.

Venegas Rodríguez, R., Hernández Pedroso, W., \& Chacón Montano, D. (2020). Ictus en pacientes geriátricos ingresados en una sala de cuidados intermedios de medicina. Revista Cubana de Medicina Militar, 49(3), e0200580. 


\section{CITAR ESTE ARTICULO:}

Borja Santillán, M. A., Samaniego Gallino, J. L., Aguirre Ruilova, S. D., \& Prieto Ulloa, M. G. (2021). Enfermedad cerebrovascular isquémica e hipertensión arterial en el hospital Teodoro Maldonado Carbo. RECIMUNDO, 5(Especial 1), 31-42. https://doi.org/10.26820/recimundo/5.(esp.1).nov.2021.31-42

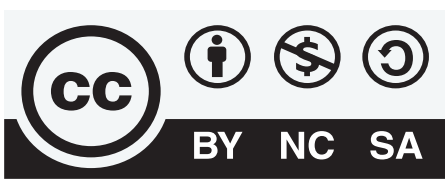

CREATIVE COMMONS RECONOCIMIENTO-NOCOMERCIAL-COMPARTIRIGUAL 4.0. 